\title{
A contribuição de Daniel Hogan para os estudos dos desastres $^{1^{*}}$
}

\author{
Thiago Fernando Bonatti ${ }^{2}$ \\ Tathiane Mayumi Anazawa
}

\begin{abstract}
Resumo: Os desastres são fenômenos sociais e seus elementos de análise precisam identificar não só suas consequências, mas também os processos históricos que fomentam sua origem. Nesse sentido, busca-se discutir o que Daniel Hogan traz para este debate, a partir de elementos relacionados às populações em situação de risco, tais como suas diferentes condições de vulnerabilidade, os aspectos demográficos, a distribuição espacial e a percepção dos riscos e perigos. O presente trabalho tem como objetivo analisar a contribuição de Hogan para a abordagem dos desastres, pela perspectiva dos estudos de População e Ambiente. Estão aqui apresentadas as discussões iniciais propostas por Daniel Hogan, os diálogos estabelecidos com outras disciplinas e três exemplos de operacionalização dos estudos sobre desastres envolvendo outros elementos de análise.
\end{abstract}

Palavras-chave: Desastres. População e Ambiente. Demografia.

\section{Daniel Hogan's contribution to disasters studies}

Abstract: Disasters are social phenomena and their elements of analysis need to identify not only their consequences, but also the historical processes that determine their origin. In this sense, the purpose is to discuss what Daniel Hogan brings to this debate, based on elements related to populations at risk, such as their different conditions of vulnerability, demographic aspects, spatial distribution and the

$1^{*}$ Os autores agradem às agências de fomento CAPES e CNPq pelo financiamento das bolsas de mestrado e doutorado.

${ }^{2}$ Mestre e doutorando em Demografia. Instituto de Filosofia e Ciências Humanas (IFCH-UNICAMP). Campinas, São Paulo. E-mail: thiagofbonatti@gmail.com.

${ }^{3}$ Doutora em Demografia. Pós-doutoranda no Instituto Nacional de Pesquisas Espaciais (INPE). São José dos Campos, São Paulo. E-mail: tathimay@gmail.com. Submetido em: 27/03/2021 - Aceito em: 17/05/2021. 
$|2|$

A contribuição de Daniel Hogan...

perception of risks and dangers. This paper aims to analyze Hogan's contribution to disasters' analyses, based on the perspective of Population and Environment studies. Here we present the initial discussions proposed by Daniel Hogan, the dialogues established with other disciplines and three examples of operationalizing studies on disasters involving other elements of analysis.

Keywords: Disasters. Population and Environment. Demography.

\section{La contribución de Daniel Hogan a los estudios de desastres}

Resumen: Los desastres son fenómenos sociales y sus elementos de análisis necesitan identificar no solo sus consecuencias, sino también los procesos históricos que determinan su origen. En este sentido, el propósito es discutir lo que Daniel Hogan aporta a este debate, a partir de elementos relacionados con las poblaciones en riesgo, como sus diferentes condiciones de vulnerabilidad, los aspectos demográficos, la distribución espacial y la percepción de riesgos y peligros. Este artículo tiene como objetivo analizar la contribución de Hogan al abordaje de los desastres, desde la perspectiva de los estudios de Población y Medio Ambiente. A continuación, se presentan las discusiones iniciales propuestas por Daniel Hogan, los diálogos establecidos con otras disciplinas y tres ejemplos de estudios operacionales sobre desastres que involucran otros elementos de análisis.

Palabras clave: Desastres. Población y Ambiente. Demografía.

\section{Introdução}

A premissa deste trabalho é que os desastres não são naturais. O desastre "es un fenómeno eminentemente social" (LAVELL THOMAS, 1993, p. 118), que necessita de uma análise do contexto de sua criação, bem como das características que o definem como tal. O impacto em um território e sua estrutura social vulnerável, o grau desse impacto e a capacidade de resposta dessa população, são pontos que devem ser considerados nos estudos dos desastres (LAVELL THOMAS, 1993).

Os desastres são abordados por diferentes áreas do conhecimento e suas definições, em sua maioria, são referentes às consequências dos desastres, e menos frequentes são os estudos que analisam as causas dos fenômenos (WILCHES-CHAUX, 
1993). Nesse contexto, enfatiza-se que incluir a dimensão social nos estudos dos desastres fez com que novos elementos fossem apresentados, para compor uma análise com maior complexidade.

Duas ideias pautam as discussões propostas por Lavell Thomas (1993), no contexto dos desastres socialmente construídos: vulnerabilidade e territorialidade. A análise da vulnerabilidade se remete também a uma dimensão temporal, ao introduzir a questão histórica de definição e redefinição dos diferentes graus de vulnerabilidade de uma população. Já a territorialidade permite a análise em diferentes territórios, desde o nível regional até a análise familiar.

Nesse sentido, Daniel Hogan introduz a perspectiva da relação População e Ambiente nos estudos sobre desastres, à luz da dinâmica demográfica. $\mathrm{O}$ autor propõe ainda as primeiras análises sobre os impactos dos desastres, ou seja, "como processos populacionais alteram o ambiente, e como fatores ambientais influenciam na dinâmica demográfica" (HOGAN, 1989, p. 72), abrindo espaço para uma nova percepção, mas que ainda não era compreendida como um problema social.

Hogan (1989) afirma que a área de estudos sobre População e Ambiente não surgiu somente da necessidade de uma produção cientifica que relacionasse tais elementos, mas sim da demanda de uma população que estava sendo afetada pelo processo de degradação ambiental, que revelam os danos causados ao ambiente decorrentes da ação humana.

Frente ao contexto apresentado, o objetivo deste trabalho consiste em analisar a contribuição do Professor Daniel Hogan para a abordagem dos desastres, pela perspectiva dos estudos de População e Ambiente. $\mathrm{O}$ artigo visa apresentar a construção de uma agenda de pesquisa dos desastres, os diálogos construídos com outros campos do conhecimento, a operacionalização dos debates em trabalhos recentes e a continuidade desta agenda frente à potencialidade dos caminhos construídos por Daniel Hogan. 
$|4|$

A contribuição de Daniel Hogan...

\section{Construção ${ }^{4}$}

Para iniciar o debate sobre os desastres pela perspectiva dos estudos de População e Ambiente, foi recuperado o trabalho desenvolvido por Hogan (1989), sobre uma série de episódios de contaminação ambiental ocorridos no período entre 1950 e 1980, que segundo o próprio autor, não foram os mais graves e nem os primeiros incidentes, mas são alguns casos que permitiram uma nova percepção sobre a degradação ambiental (HOGAN, 1989), e de certa forma moldaram a percepção sobre a relação entre População e Ambiente.

Esses eventos demonstram as transformações da análise sobre os desastres ao longo do tempo e suas implicações em termos ambientais, econômicos, sociais e demográficos, ajudando a compreender o caminho percorrido para que fosse possível analisar os eventos contemporâneos. Evidenciam-se ainda os fatos decorrentes dos desastres aqui abordados, nos âmbitos acima citados, de modo a verificar quais medidas foram tomadas frente aos impactos causados por esses eventos, quais danos causaram e, sendo este o caso, de que maneira contribuíram para o debate sobre o tema.

Para que seja possível uma análise dos casos de contaminação recentemente identificados, é fundamental ter a dimensão de como foram realizados os primeiros estudos sobre acidentes ambientais, a repercussão que tiveram e como se deu o encaminhamento da questão dos danos provocados por esses incidentes, frente à população afetada e à opinião pública, e de que maneira foram tomadas as devidas providencias para a resolução desse tipo de problema.

Neste sentido, em diversos casos (ANAZAWA 2017; BONATTI 2017; ANAZAWA; BONATTI; CARMO, 2016) demonstram-se a inoperância dos agentes responsáveis pela causa e monitoramento dos danos ambientais, assim como nos

${ }^{4}$ Parte dessa discussão é apresentada por Bonatti (2017) e Bonatti e Carmo (2015). 
episódios apresentados por Hogan (1989), no que se refere às medidas preventivas, na avaliação da exposição da população aos riscos iminentes, por vezes causados pelas atividades previamente desenvolvidas nos lugares afetados, e ainda a reutilização desses espaços para fins habitacionais, entre outros problemas. Verifica-se ainda que determinados eventos e desastres ambientais, mesmo que numa escala menor que os desastres referidos por Hogan (1989), contribuem para uma mudança na percepção em relação aos danos ambientais, bem como atentam para a atuação dos diversos agentes envolvidos nos processos de uso e ocupação dos espaços urbanizados, e as consequências do acelerado processo de expansão urbana e industrial, especialmente nos últimos quarenta anos.

Os casos resumidamente listados abaixo (Tabela 1), relacionados e analisados por Hogan (1989) são somente alguns exemplos, dentre tantos outros que poderiam estar presentes neste trabalho. Foram selecionados pela importância que tiveram frente às discussões sobre a relação entre população e ambiente, e também pela disponibilidade de estudos que identificaram a forma pela qual ocorreram, quais medidas mitigadoras foram tomadas, quais as consequências observadas para a população e de que maneira acabaram por contribuir com a evolução dos processos de monitoramento e prevenção dos riscos provenientes das atividades industriais. 
Tabela 1. Casos de contaminação por local, ano, tipo de desastre, impactos e seu legado.

\begin{tabular}{|c|c|c|c|c|}
\hline Local & Ano & $\begin{array}{l}\text { Tipo de } \\
\text { desastre }\end{array}$ & Impactos & Legado \\
\hline $\begin{array}{l}\text { Donora } \\
\text { (EUA) }\end{array}$ & 1948 & $\begin{array}{l}\text { Poluição } \\
\text { atmosférica }\end{array}$ & $\begin{array}{l}20 \text { mortos e mais } \\
\text { de } 7000 \text { pessoas } \\
\text { contaminadas }\end{array}$ & $\begin{array}{l}\text { Criação das pri- } \\
\text { meiras leis sobre } \\
\text { controle da } \\
\text { poluição }\end{array}$ \\
\hline $\begin{array}{l}\text { Londres } \\
\text { (Reino } \\
\text { Unido) }\end{array}$ & 1952 & $\begin{array}{l}\text { Inversão tér- } \\
\text { mica }\end{array}$ & 4000 mortes & $\begin{array}{l}\text { Impulsionou os } \\
\text { primeiros estudos } \\
\text { epidemiológicos } \\
\text { sobre poluição } \\
\text { do ar }\end{array}$ \\
\hline $\begin{array}{l}\text { Minamata } \\
\text { (Japão) }\end{array}$ & 1956 & $\begin{array}{l}\text { Envenena- } \\
\text { mento das } \\
\text { águas por me- } \\
\text { tais pesados }\end{array}$ & $\begin{array}{c}798 \text { casos oficiais, } \\
107 \text { mortes, } 2.800 \\
\text { casos relaciona- } \\
\text { dos }\end{array}$ & $\begin{array}{l}\text { Levantou dis- } \\
\text { cussões sobre } \\
\text { a liberação de } \\
\text { dejetos industriais } \\
\text { no ambiente, } \\
\text { principalmente o } \\
\text { mercúrio }\end{array}$ \\
\hline $\begin{array}{l}\text { Love } \\
\text { Canal } \\
\text { (EUA) }\end{array}$ & 1970 & $\begin{array}{l}\text { Despejo de } \\
\text { resíduos no } \\
\text { ambiente }\end{array}$ & $\begin{array}{c}\text { Abortos espon- } \\
\text { tâneos, defeitos } \\
\text { congênitos, câncer } \\
\text { etc. }\end{array}$ & $\begin{array}{l}\text { Criação de legis- } \\
\text { lação ambiental } \\
\text { que prevê o es- } \\
\text { clarecimento da } \\
\text { população sobre } \\
\text { a atividade das } \\
\text { industrias }\end{array}$ \\
\hline $\begin{array}{l}\text { Seveso } \\
\text { (Itália) }\end{array}$ & 1977 & $\begin{array}{l}\text { Nuvem tóxica } \\
\text { (Dioxina) }\end{array}$ & $\begin{array}{l}\text { Mortalidade por } \\
\text { problemas cardía- } \\
\text { cos, câncer, mu- } \\
\text { danças na razão } \\
\text { de sexo }\end{array}$ & $\begin{array}{c}\text { O Estudo foi es- } \\
\text { tendido até 1996, } \\
\text { para acompanha- } \\
\text { mento }\end{array}$ \\
\hline $\begin{array}{l}\text { Bhopal } \\
\text { (Índia) }\end{array}$ & 1984 & $\begin{array}{l}\text { Vazamento de } \\
\text { gás venenoso }\end{array}$ & $\begin{array}{c}2.500 \text { mortes e } \\
50.000 \text { afetados }\end{array}$ & $\begin{array}{l}\text { Maior atenção so- } \\
\text { bre a exportação } \\
\text { de produtos quí- } \\
\text { micos perigosos }\end{array}$ \\
\hline $\begin{array}{l}\text { Chernobyl } \\
\text { (URSS } \\
\text {-Ucrânia) }\end{array}$ & 1986 & $\begin{array}{l}\text { Explosão e } \\
\text { liberação de } \\
\text { material ra- } \\
\text { dioativo }\end{array}$ & $\begin{array}{l}4000 \text { mortes e } \\
600.000 \text { contami- } \\
\text { nados }\end{array}$ & $\begin{array}{l}\text { Monitoramento } \\
\text { da produção de } \\
\text { energia e de resí- } \\
\text { duos nucleares }\end{array}$ \\
\hline
\end{tabular}

Fonte: HOGAN (1989). 
Esses incidentes se tornaram emblemáticos para uma nova percepção, ainda que não tenham sido considerados até então como um problema social. Ocorreram num contexto de favorável comunicação, que tornou possível o acompanhamento de desastres locais por todo o mundo, e segundo Hogan (1989), se deram em contextos de "maturidade" e independência das instituições de saúde, possibilitando investigações aprofundadas sobre estes casos ocorridos. $\mathrm{O}$ autor destaca que os eventos eram tratados de maneira singular, sem serem relacionados a outros eventos semelhantes, no contexto dos problemas ambientais, mas deram início a ações e publicações que buscavam relacionar os desastres ocorridos. Aponta ainda que esses desastres, mesmo que tenham gerado discussões no âmbito ambiental, não questionavam a "ideologia e o progresso da tecnologia" (HOGAN, 1989), sendo analisados como parte do processo de desenvolvimento, e os trabalhos realizados para combater a contaminação, como um modelo de "resposta" a "novos desafios" impostos pela modernização. A própria atitude dos setores públicos e privados girava em torno de negar o ocorrido e de acalmar a população. Não havia planos de avaliação dos desastres e nem de emergência, e o cenário que se reproduzia era o das indústrias negando responsabilidade e restringindo informações sobre os eventos.

O debate populacional girava em torno da "explosão demográfica", e no contexto da relação entre População e Ambiente, o que se observava era a crescente percepção da interação entre saúde e "fatores de degradação ambiental", dando destaque para a preocupação dos cientistas sobre as implicações das contaminações do ar e da água sobre a saúde. Para Hogan (1989) a primeira resposta da Demografia para a questão foi a "equação malthusiana" ${ }^{5}$, sendo

\footnotetext{
${ }^{5}$ A questão malthusiana e sua importância para os estudos de população e ambiente se faz presente, no início dessas discussões, sob a forma da pressão dos números sobre os recursos, como temas relacionados à desertificação, fome, esgotamento de recursos, entre outros (HOGAN, 1991). Partindo de uma revisão sobre a constituição do campo de População e Ambiente, D'Antona (2017) indica que esta é realizada a partir de dois mitos: o malthusiano e o das
} 
$|8|$

A contribuição de Daniel Hogan...

as questões ambientais consequência da "explosão demográfica". Havia, contudo, uma ala de demógrafos progressistas, que viam o crescimento demográfico como um dos fatores agravantes dos problemas ambientais, mas não como a causa destes.

As consequências das "doenças ambientais" para a fecundidade e a mortalidade não foram reconhecidas naquele momento pelos demógrafos, não havendo percepção de uma nova modalidade de análise. Nesse cenário, o surgimento das questões ambientais colocava em xeque os avanços tecnológicos a partir da observação dos casos citados anteriormente, e dos procedimentos adotados para reparar os danos causados. Muitas vezes, as atitudes tomadas visavam reparar problemas através da substituição dos agentes causadores das doenças, desenvolvendo um aparato tecnocrata de análise e saneamento.

Após vários acidentes graves ocorridos na década de 1980, e confirmando a necessidade de conscientização sobre os riscos tecnológicos frente às pessoas e ao ambiente, a ONU declarou que a década de 1990 seria a "Década Internacional para Redução dos Desastres". Promovendo a reorganização de órgãos internacionais de gestão de emergências, criou o Departamento de Assuntos Humanitários, visando uma atuação mais direta e efetiva em relação aos casos de desastres. E muito embora o direcionamento fosse maior em relação aos desastres naturais, a partir da metade dos anos 1990, foram incluídos também os desastres tecnológicos no campo de ação. A aproximação dos desastres naturais com os desastres tecnológicos, ampliou o escopo da discussão sobre componentes, causas e consequências desse tipo de desastre, possibilitando o aprofundamento sobre a temática e melhorando as condições de análise desenvolvidas.

Além da análise de casos de contaminação de repercussão internacional, outro trabalho de Hogan (1993), fundamental para essa discussão, é o estudo desenvolvido pelo autor sobre a cidade de Cubatão, conhecida nos anos 1980 por ser um dos principais polos petroquímicos do país, mas que também ficou marcado

relações recíprocas. 
pelos problemas ambientais relacionados à "chuva ácida, defeitos congênitos, desflorestamento, e a poluição do ar, água e solo" (HOGAN, 1993), um dos grandes exemplos de como se deu o crescimento industrial sem levar em consideração o cuidado com o ambiente.

Hogan analisa diversos aspectos demográficos relacionados à população de Cubatão, como o fenômeno da migração pendular, dados sobre seletividade, cor, estado civil, região de origem, ocupação, de indicações socioeconômicas, como empregos formais e informais, taxa de escolaridade, segregação residencial entre outros indicadores, aproximando mais ainda o escopo demográfico das questões ambientais, um passo fundamental para sedimentar os estudos de População e Ambiente como uma vertente sólida dos estudos populacionais. Dentre os aspectos que chamam a atenção, está a questão da percepção ambiental da poluição, que no caso de Cubatão, segundo a própria população residente, era somente característica da zona industrial do município, e não das zonas residenciais, e por conta disso, os esforços em torno de chamar atenção para o problema tinham que ser não somente direcionados às indústrias e ao governo, mas também aos diversos segmentos populacionais do município (HOGAN, 1993).

\section{Diálogos $^{6}$}

Os desastres são amplamente discutidos pela literatura desde o início do século XX, com intensificação das pesquisas sobre desastres após a Segunda Guerra Mundial, conforme apresentado por Dynes e Drabek (1994), e também por Quarantelli e Dynes (1977). Contudo, as abordagens sobre as discussões dos desastres são pautadas em diferentes disciplinas e escopos teóricos, marcados pela determinação de um desastre como evento ou processo. Caracterizar o desastre com tal, evento ou processo, implica reposicionar o próprio conceito de desastre.

\footnotetext{
${ }^{6}$ Parte dessa discussão é apresentada por Anazawa (2017).
} 
Segundo Valencio (2014), nos estudos sobre desastres, há que se diferenciar a teoria dos hazards e a teoria dos desastres. Enquanto a primeira está relacionada com a abordagem geográfica, a teoria dos desastres encontra sua base na abordagem sociológica. A teoria dos hazards tem seu foco nos mecanismos físicos do desastre, bem como a distribuição temporal e espacial dos eventos. As medidas utilizadas nessa teoria estão relacionadas à magnitude, frequência e duração dos desastres (MATEDDI; BUTZKE, 2001). Já a teoria dos desastres tem seu foco voltado para a dimensão humana dos desastres, ou seja, para a organização social complexa e o comportamento coletivo (VALENCIO, 2014).

Segundo Lavell Thomas e Franco (1996), tratar o desastre como evento é posicioná-lo como imprevisível, ingovernável e inevitável. Os autores também enfatizam que as ciências da terra e as engenharias, ao se consolidarem como disciplinas, fortaleceram os estudos dos desastres como eventos. Como resposta a esse recorte teórico, os estudos tiveram foco na prevenção e previsibilidade dos desastres, uma vez que os eventos físicos foram considerados os principais responsáveis dos desastres e por serem considerados anormais e imprevisíveis. Essa mesma visão do desastre como inevitável e incontrolável, apresenta a questão da organização da sociedade em função ao enfrentamento do desastre e a forma de conduzir a mesma ao seu estado de "normalidade" (LAVELL THOMAS; FRANCO, 1996).

O caráter pontual utilizado para a definição do desastre engessa o entendimento do mesmo como um processo. Diante desse cenário, não há discussões sobre as relações sociais que potencializam a vulnerabilidade das pessoas que residem em área de risco (VALENCIO, 2009).

Cabe então, discutir e definir os desastres baseados na abordagem social, cujos estudos têm início na década de 1940 e ganham força e estrutura a partir de 1960. O pioneiro é Charles Fritz, que introduz o conceito de desastre socialmente construído em 1961 (MASKREY, 1993). Em seguida, ganha espaço a corrente teórica norte-americana, tendo como um dos representantes expressivos, Enrico Quarantelli, cuja obra "O que é um desastre?" 
(1998), representa um marco importante para a abordagem social. Esta obra indica que o desastre, a partir da abordagem social, pode ser configurado como um processo. Contudo, essa corrente é marcada por um enfoque próprio da sociologia norte-americana e seus estudos empíricos (MASKREY, 1993). Na América Latina, os estudos sobre a abordagem social dos desastres ganham destaque no final da década de 1980, e são concentrados pela Red de Estudios Sociales en Prevención de Desastres en América Latina (LA RED), formada em 1992. O marco teórico publicado pela LA RED consiste na obra organizada por Maskrey (1993), intitulada como "Los desastres no son naturales". No capítulo apresentado por Wilches-Chaux (1993), o posicionamento do desastre como um processo torna-se claro a partir da análise contextualizada na Teoria dos Sistemas.

A partir da abordagem sociológica dos desastres, destacamse os estudos realizados no Brasil, especificamente do Núcleo de Estudos e Pesquisas Sociais em Desastres (NEPED)/UFSCAR, evidenciando os trabalhos de Norma Valencio e a abordagem da construção social dos desastres (VALENCIO et al., 2009; VALENCIO, 2010; 2013; VALENCIO; SIENA, 2014).

A abordagem pela perspectiva social dos desastres permite também o diálogo com outras temáticas referentes à população atingida. Nesse sentido, o próprio campo de População e Ambiente se faz presente ao propor o diálogo com as ciências sociais e as ciências ambientais (D'ANTONA, 2017), incluindo diferentes temáticas que propõe a reflexão da questão dos desastres sob a perspectiva social. Analisando a evolução dos trabalhos no campo de População e Ambiente, cujos precursores no Brasil foram Martine, Sawyer e Hogan, D’Antona (2017, p. 261) resgata as temáticas envolvidas: “Nos anos 1980-90, os principais trabalhos incluíam os referenciais de capacidade de suporte, sustentabilidade e desenvolvimento sustentável; nas décadas seguintes, incorporaram-se os de vulnerabilidade e risco".

Ao analisar os estudos sobre população e ambiente desde a criação do Grupo de Trabalho População e Ambiente, na ABEP, em 1990, Marandola Jr. e Hogan (2007) indicam que os temas riscos e 
vulnerabilidade, cidade e degradação social e ambiental e conflito população-recursos no enfoque regional, foram consolidados como grandes temas desde meados dos anos 90. Os autores consideram que dois eixos temáticos se tornaram mais amplos em meio aos estudos de população e ambiente: situações de risco e vulnerabilidade. Trabalhar com a questão do risco é proporcionar uma leitura diferenciada sobre a forma como se encaram os conflitos existentes entre população, recurso e Estado. Em outro momento, Marandola Jr. e Hogan (2004) apresentam a discussão e os avanços proporcionados pelo diálogo entre as ciências sociais e a geografia, para os estudos de riscos e perigos.

Marandola Jr. e Hogan (2006) enfatizam que na área de População e Ambiente e seu diálogo com as ciências sociais, encontram-seasleiturasintegradasdasdimensõessocialeambiental. Torres (2000), em busca por uma demografia do risco ambiental, afirma que uma abordagem sociológica e demográfica da questão ambiental, permite a identificação de diferentes características sociais e demográficas de diversos grupos populacionais, expostos a diferenciados graus de riscos ambientais. A demografia se encontra desafiada nesta temática por que precisa pensar em uma escala intraurbana (demografia de pequenas áreas), uma vez que a demografia convencional tenha se atentado a processos macro, como o crescimento e distribuição da população e os componentes da dinâmica demográfica (mortalidade, fecundidade e migração).

A dinâmica demográfica caracterizada pela composição da população em termos de sexo e idade, consiste em uma importante base para a compreensão da construção social dos desastres. Essas características demográficas auxiliam na compreensão dos desastres (CARMO, 2015; CARMO; ANAZAWA, 2014). Além disso, há a necessidade de analisar a evolução da população não só em termos de volume, mas também sua composição etária, que determina, por exemplo, quais as características da população mais vulnerável aos desastres.

Além de conhecer as características da população, é importante analisar como ocorreu sua redistribuição espacial ao longo do tempo, configurando situações de vulnerabilidade 
e não acessibilidade (CARMO, 2015). Nesse sentido, destacamse os processos migratórios e a intensa e rápida urbanização brasileira, que resulta na concentração da população em diferentes localidades espaciais, com diversas formas de ocupação, revelando assim diferentes graus de exposição dos riscos dos desastres.

Segundo Carmo (2015), nos estudos sobre desastres, há que se considerar a distribuição espacial da população sob duas perspectivas: a forma com que a população ocupa historicamente o espaço pode levar a situações de risco do desastre, como também as características dessa distribuição, podendo ocupar áreas com maior ou menor dificuldade de acesso, implicando nas situações pós-desastre, como por exemplo, o socorro. Essas perspectivas ficam claras ao se pensar em um desastre de grande impacto, como é o caso de enchentes e deslizamentos: as populações que ocupam áreas próximas a corpos d'água e em locais com alta declividade, encontram-se mais vulneráveis aos desastres relacionados. No entanto, no caso das secas e estiagens, a correlação direta entre essas características não ocorre da mesma forma, como o presente trabalho buscará mostrar.

Para se trabalhar com os estudos da população em situação de risco, sob o olhar da demografia, uma série de desafios metodológicos e empíricos deve ser considerada, como a questão da escala dos processos e a integração de dados sociodemográficos e ambientais.

Segundo Torres (2000), ainda há outra complicação, uma vez que estudos desse tipo colocam em evidência os problemas relativos ao tamanho da população e sua distribuição, em relação aos componentes da dinâmica demográfica. O que significa dizer que outras variáveis devem ser incorporadas ao estudo da demografia clássica, como por exemplo, as variáveis socioeconômicas da população e as variáveis relacionadas a questão da moradia.

No entanto, para o estudo dos desastres, não é preciso solidificar uma "unificação de linguagem" (MARANDOLA Jr.; HOGAN, 2004, p. 106) ou "la construcción de un objeto nuevo de estudio, en uma teoría de los desastres, o en una ciencia de la 'desastrología' " (LAVELL THOMAS, 1993, p.123). Enfatiza- 
se que a busca seja pelo diálogo entre os diferentes campos do conhecimento, releituras de conceitos e integração de metodologias quali-quantitativas.

\section{Operacionalização}

Para apresentar a operacionalização da discussão sobre a abordagem dos desastres, pela perspectiva dos estudos de População e Ambiente, foram selecionados três trabalhos, em diferentes áreas de estudo, analisando contextos diferentes de desastres, mas que carregam similaridades em sua forma de operacionalização do conceito de desastre e sua construção.

O primeiro trabalho consiste em uma dissertação, intitulada como "Expansão urbana e a ocupação de áreas contaminadas: o caso do bairro Mansões Santo Antônio em Campinas, São Paulo". Bonatti (2017), utiliza-se do pensamento proposto por Hogan para analisar um caso específico de contaminação do solo, ocorrido no Município de Campinas, São Paulo. O autor apresenta os problemas decorrentes deste desastre, porém sob a ótica dos estudos demográficos, atentando também para os aspectos socioeconômicos que interagem com este problema. São considerados na análise a expansão desordenada do tecido urbano, a reutilização de áreas contaminadas, que em um primeiro momento haviam sido destinadas à atividade industrial, e as características sociodemográficas da população residente, que se diferencia das características frequentemente encontradas em populações expostas aos danos e riscos ambientais. $\mathrm{O}$ referido caso está situado em um bairro de classe média/alta, em um dos espaços de maior procura e desenvolvimento recente no município de Campinas, devido à sua localização, próxima às principais rodovias que interligam o município à região e ao centro da cidade. Desse modo, o autor busca integrar a discussão ambiental, em torno do desastre ocorrido, em razão de atividade industrial, à dinâmica populacional, através da distribuição espacial da população e de suas características sociodemográficas. 
O segundo trabalho é referente a tese intitulada "A grave escassez hídrica e as dimensões de um desastre socialmente construído: a região metropolitana de Campinas entre 20132015", cujo objetivo foi realizar a leitura da escassez hídrica ocorrida especificamente na região sudeste, entre 2013 e 2015, como um desastre socialmente construído (ANAZAWA, 2017). A autora propõe uma matriz conceitual baseada na relação vulnerabilidade-segurança humana no contexto da segurança hídrica, que permitiu analisar elementos para a compreensão da relação População e Ambiente. A operacionalização desta matriz foi a partir de diferentes formas de representação para três dimensões: a Dimensão Institucional, a Dimensão da População e seus Territórios e a Dimensão da Percepção. Foram abordados neste trabalho, elementos como a questão espacial e multiescalar do fenômeno, a construção histórica da escassez hídrica entendida enquanto um processo, as questões institucionais envolvidas no uso e gestão do recurso hídrico e as características da população e suas diferentes respostas ao desastre a partir de dados secundários e dados primários obtidos a partir de pesquisa de campo, cuja finalidade foi analisar as diferentes percepções sobre o desastre, especificamente no município de Campinas, entre 2013 e 2015.

O terceiro e último refere-se ao trabalho apresentado no VII Congresso da Associação Latino-Americana de População e o XX Encontro Nacional de Estudos Populacionais: “O risco construído: reflexões sobre o desastre ocorrido em Mariana, estado de Minas Gerais, em 2015, a partir da perspectiva da relação entre população e ambiente". O objetivo deste trabalho foi revisitar o conceito de desastre e analisar o rompimento da barragem de rejeitos de mineração de Fundão, localizada no município de Mariana, em Minas Gerais, ocorrido em 5 de novembro de 2015 e considerado um dos maiores desastres ambientais da história brasileira (ANAZAWA; BONATTI; CARMO, 2016). O trabalho questiona as diferenças nas definições dos desastres enquanto naturais e tecnológicos, e suas implicações frente ao enfrentamento do desastre. Segundo os autores, no caso do desastre de Mariana a construção social do desastre é evidente, por trazer elementos 
históricos relacionados aos problemas que a barragem já apresentava, bem como a localização de população em situação de risco próximo à barragem. São considerados elementos como a análise histórica referente ao problema das barragens, outros desastres ocorridos no estado de Minas Gerais e a distribuição da população potencialmente impactada pelo desastre.

Foi possível observar, ao apresentar os três trabalhos, como diferentes elementos foram inseridos na discussão dos desastres pela perspectiva da relação População e Ambiente, buscando analisar não apenas o impacto causado pelos diferentes desastres, mas sua construção histórica e principalmente as populações em situação de risco, em diversas condições de vulnerabilidade e que apresentam percepções e respostas distintas.

\section{Continuidade: para não concluir}

O propósito de recuperar os trabalhos aqui apresentados, especialmente as questões levantadas por Daniel Hogan, fora atentar para a continuidade e a influência de seu pensamento nos estudos sobre os desastres. Ainda que os desastres sigam acontecendo, por vezes de maneira distinta às estudadas até então, as perspectivas analíticas em torno dos desastres e da relação com a população e o ambiente também vão se alterando e se complexificando, auxiliando na sua identificação dos eventos adversos, levantando novas questões e demandas, e evidenciando os caminhos a serem percorridos e observados, com propósito de acompanhar as distintas dinâmicas populacionais e as questões pertinentes a elas.

Pautar-se pelo que Daniel Hogan desenvolveu durante anos de pesquisa é fundamental para que haja conhecimento de todo o percurso do pesquisador, especialmenteno que se refere à busca pela integração das questões relacionadas à população e ao ambiente. Os exemplos de desastres por ele analisados, e neste trabalho sucintamente retomados, demonstram como tem sido a evolução dos estudos sobre os desastres, e das possibilidades analíticas que têm se desenvolvido desde então. Talvez o principal ponto 
a ser absorvido pela produção teórica desenvolvida por Daniel Hogan, tenha sido a busca pelo diálogo entre as diversas áreas do conhecimento, especialmente sobre as questões demográficas, que extrapolam o núcleo duro desta área do conhecimento, trazendo novas possibilidades de captação de informações e de análises por meio de elementos que antes seriam restringidos a uma ou outra disciplina.

Carmo e Valencio (2014) apontam para um desafio da atualidade em abordar os temas vulnerabilidade e desastres na produção científica, buscando complexificar o debate a partir da caracterização das "múltiplas dimensões da vulnerabilidade e as diferentes facetas dos recorrentes desastres" (CARMO; VALENCIO, 2014, p. XV). O diálogo já se faz presente nos estudos sobre desastres, principalmente entre a Geografia, Demografia e Sociologia Ambiental. No entanto, é na Demografia que se busca o maior diálogo para proporcionar uma leitura multidimensional do fenômeno (MARANDOLA JR.; HOGAN, 2004).

O debate proposto por Daniel Hogan segue atual, mesmo após trinta anos das publicações aqui apresentadas, visto que os desastres seguem ocorrendo, de maneiras cada vez mais específicas, despertando a necessidade recorrente de compreendêlos e buscando por elementos que possibilitem a sua mitigação. Tendo sido elaborados em um período de grande crescimento tecnológico e industrial, de expansão dos espaços urbanos no Brasil, os estudos de Daniel Hogan abriram caminhos e tornaramse base para o estudo uma temática importante e crescente, que são os estudos dos desastres, em suas distintas vertentes, bem como o aprofundamento das questões sobre a população e o ambiente, pautado pela dinâmica populacional. 
$|18|$

A contribuição de Daniel Hogan...

\section{Referências}

ANAZAWA, Tathiane Mayumi. A grave escassez hídrica e as dimensões de um desastre socialmente construído: a Região Metropolitana de Campinas entre 2013-2015. 2017. 369 p. Tese (doutorado em Demografia) - Instituto de Filosofia e Ciências Humanas, Universidade Estadual de Campinas, Campinas, São Paulo, 2017.

ANAZAWA, Tathiane Mayumi; BONATTI, Thiago Fernando; CARMO, Roberto Luiz do. O risco construído: reflexões sobre o desastre ocorrido em Mariana, estado de Minas Gerais, em 2015, a partir da perspectiva da relação entre população e ambiente. In: VII Congreso de la Asociación Latinoamericana de Población e XX Encontro Nacional de Estudos Populacionais, 2016, Foz do Iguaçu. Anais [...]. Foz do Iguaçu: ABEP/ALAP, 2016.

BONATTI, Thiago Fernando. Expansão urbana e a ocupação de áreas contaminadas: o caso do bairro Mansões Santo Antônio em Campinas, São Paulo. 2017. 152 p. Dissertação (mestrado em Demografia) - Instituto de Filosofia e Ciências Humanas, Universidade Estadual de Campinas, Campinas, São Paulo, 2017.

BONATTI, Thiago Fernando; CARMO, Roberto Luiz do. Bairro Mansões Santo Antônio em Campinas, São Paulo: Expansão Urbana, Contaminação do Solo, Ocupação Desordenada e Danos à Saúde. In: VII Encontro Nacional da ANPPAS, 2015, Brasília. Anais [...]. Brasília: ANPPAS, 2015.

CARMO, Roberto Luiz do. População, riscos, vulnerabilidade e desastres: conceitos básicos. In: SIQUEIRA, Antenora; VALENCIO, Norma; SIENA, Mariana; MALAGOLI, Marco (Org.). Riscos de desastres relacionados à água: aplicabilidade das bases conceituais das Ciências Humanas e Sociais na análise de casos concretos. São Carlos: RiMA, 2015.

CARMO, Roberto Luiz do; ANAZAWA, Tathiane Mayumi. Mortalidade por desastres no Brasil: o que mostram os dados. Ciência \& Saúde Coletiva, v. 19, n. 9, p. 3669-3681, set. 2014. 
CARMO, Roberto Luiz do; VALENCIO, Norma. Apresentação. In: CARMO, Roberto Luiz do; VALENCIO, Norma. (Org.). Segurança Humana em contextos de desastres. São Carlos: Editora Rima, p. xv-Xvii, 2014.

D'ANTONA, Álvaro de Oliveira. Do mito malthusiano ao das relações recíprocas - a constituição interdisciplinar do campo de População e Ambiente. Rev. bras. estud. popul., São Paulo, v. 34, n. 2, p. 243-270, 2017.

DYNES, Russell; DRABEK, Thomas. The structure of disaster research: its policy and disciplinary implications. International Journal of Mass Emergencies and Disasters, v.12, n.1, p. 5-23. 1994.

HOGAN, Daniel Joseph. Crescimento demográfico e meio ambiente. Revista Brasileira de Estudos de População, v. 8, n 1/2, 1991.

HOGAN, Daniel Joseph. População e Meio-Ambiente - Texto Nepo 16. Campinas: NEPO/ UNICAMP - Textos NEPO, 1989.

HOGAN, Daniel Joseph. População, Pobreza e Poluição em Cubatão, São Paulo. In: MARTINE, George (Org.). População, Meio Ambiente e Desenvolvimento: verdades e contradições. Campinas: Ed. da Unicamp, 1993. p. 101-131.

LAVELL THOMAS, Allan. Ciencias Sociales y Desastres Naturales en America Latina: Un Encuentro Inconcluso. In: MASKREY, Andrew (Org.). Los Desastres no son Naturales. LaRED - Red de Estudios Sociales en Prevencion de Desastres en America Latina, 1993.

LAVELL THOMAS, Allan; FRANCO, Eduardo (Org.). Estado, Sociedad y Gestión de los Desastres en América Latina. En busca del paradigma perdido. Lima, Perú: La Red-FLACSO-IT. Perú. Lahmann. 1996.

MARANDOLA JR., Eduardo; HOGAN, Daniel Joseph. As dimensões da vulnerabilidade. São Paulo em Perspectiva, v. 20, p. 33-43, 2006. 
$|20|$

A contribuição de Daniel Hogan...

MARANDOLA JR., Eduardo; HOGAN, Daniel Joseph. Em direção a uma demografia ambiental? Avaliação e tendências dos estudos de população e ambiente no Brasil. Rev. bras. estud. popul. v.24, n.2., p. 191-223, 2007.

MARANDOLA JR., Eduardo; HOGAN, Daniel Joseph. Natural hazards: o estudo geográfico dos riscos e perigos. Ambiente e Sociedade, Campinas, v. 7, n.2, p. 95-110, 2004.

MASKREY, Andrew (Org.). Los Desastres no son Naturales. LaRED - Red de Estudios Sociales en Prevencion de Desastres en America Latina, 1993.

MATTEDI, Marcos Antônio; BUTZKE, Ivani Cristina. A relação entre o social e o natural nas abordagens de hazards e desastres. Ambiente \& Sociedade, n. 9, p. 1-16, 2001.

QUARANTELLI, Enrico (Ed.). What is a Disaster?. London: Routledge, 1998.

QUARANTELLI, Enrico; DYNES, Russell. Response to social crisis and disaster. Annual Review of Sociology, n. 3, p. 23-49, 1977.

TORRES, Haroldo da Gama. A demografia do risco ambiental. In: TORRES, Haroldo da Gama; COSTA, Heloísa (Org.). População e meio ambiente: debates e desafios. São Paulo: Senac, 2000. p. 53-73.

VALENCIO, Norma. Da 'área de risco' ao abrigo temporário: uma análise dos conflitos subjacentes a uma territorialidade precária. In: VALENCIO, Norma; SIENA, Mariana; MARCHEZINI, V.; GONÇALVES, Juliano Costa (Org.). Sociologia dos Desastres: construção, interfaces e perspectivas no Brasil, Volume I. São Carlos: Rima Editora, 2009.

VALENCIO, Norma. Desastres: tecnicismo e sofrimento social. Ciênc. saúde coletiva, v. 19, n. 9, p. 3631-3644, 2014.

VALENCIO, Norma. Sociologia dos Desastres: construção, interfaces e perspectivas no Brasil, Volume II. 1. ed. São Carlos: RiMa Editora, 2010. v. II. 248p. 
VALENCIO, Norma. Sociologia dos desastres: construção, interfaces e perspectivas no Brasil, Volume III. São Carlos: RiMa Editora, 2013, v.III.

VALENCIO, Norma; SIENA, Mariana; MARCHEZINI, V.; GONÇALVES, Juliano Costa (Org.). Sociologia dos Desastres: construção, interfaces e perspectivas no Brasil, Volume I. São Carlos: Rima Editora, 2009.

VALENCIO, Norma; SIENA, Mariana (Org.). Sociologia dos Desastres: construção, interfaces e perspectivas, Volume IV. São Carlos: RiMa Editora, 2014. v. IV. 379p.

WILCHES-CHAUX, Gustavo. La Vulnerabilidad Global. In: MASKREY, Andrew (Org.). Los Desastres no son Naturales. LaRED - Red de Estudios Sociales en Prevencion de Desastres en America Latina, 1993. 